\title{
TOPOLOGICAL ENTROPY OF FIXED-POINT FREE FLOWS
}

\author{
ROMEO F. THOMAS
}

\begin{abstract}
Topological entropy was introduced as an invariant of topological conjugacy and also as an analogue of measure theoretic entropy. Topological entropy for one parameter flows on a compact metric spaces is defined by Bowen. General statements are proved about this entropy, but it is not easy to calculate the topological entropy, and to show it is invariant under conjugacy. For all this I would like to try to pose a new direction and study a definition for the topological entropy that involves handling the technical difficulties that arise from allowing reparametrizations of orbits. Some well-known results are proved as well using this definition. These results enable us to prove some results which seem difficult to prove using Bowen's definition. Also we show here that this definition is equivalent to Bowen's definition for any flow without fixed points on a compact metric space. Finally, it is shown that the topological entropy of an expansive flow can be defined globally on a local cross sections.
\end{abstract}

\section{INTRODUCTION}

Topological entropy was introduced as an invariant of topological conjugacy and also as an analogue of measure theoretic entropy. Topological entropy for one parameter flows on compact metric spaces is defined by Bowen in [1, 2]. General statements are proved about this entropy, but it is not easy to calculate the topological entropy, and to show it is invariant under conjugacy. For all this I would like to try to pose a new direction and study a definition for the topological entropy that involves handling the technical difficulties that arise from allowing reparametrizations of orbits and to clarify some results which are stated in the literature without proof. This definition has been introduced in [13]. Thus we consider the first part of this work as a continuation of [13]. In $\S 2$ an illustrative example is given. In $\S 3$ we prove that this definition is equivalent to Bowen's definition for any flow without fixed points on a compact metric space (i.e., we prove the conjecture raised in [13]). In $\S 5$ we investigate an important question of whether the topological entropy can by defined globally on local cross sections. Theorem 34 in $\S 5$ is a general result in this direction.

Received by the editors August 22, 1988.

1980 Mathematics Subject Classification (1985 Revision). Primary 58F25, 54C70; Secondary $43 \mathrm{C} 35,58 \mathrm{~F} 15$.

Key words and phrases. Flows, expansive, $h$-expansive, nonwandering sets, conjugacy, Axiom $A$, local cross sections. 


\section{Preparatory definitions and lemmas}

In this paper we assume that the spaces are compact metric spaces unless otherwise stated, and $(X, \varphi)$ denotes a continuous real flow $\varphi$ on a compact metric space $X$ (i.e., $\varphi: X \times R \rightarrow R$ is continuous and $\varphi(x, t+s)=\varphi(\varphi(x, t), s)$ ). Let $\varphi_{t}$ denote the homeomorphism of $X$ defined by $\varphi_{t} x=\varphi(x, t)$.

We now review the definition of topological entropy given in $[1,3]$.

For $E, F \subseteq X$ we say that $E(t, \delta)$-spans $F$ (with respect to $\varphi$ ), if for every $x \in F$ there is an $e \in E$ so that $d\left(\varphi_{s} e, \varphi_{s} x\right) \leq \delta$ for all $0 \leq s \leq t$. Let $r_{t}(F, \delta)=r_{t}(F, \delta, \varphi)$ denote the minimum cardinality of a set which $(t, \delta)$ spans $F$. If $F$ is compact, then continuity of $\varphi$ guarantees $r_{t}(F, \delta)<\infty$. We define

$$
h(\varphi \mid F, \delta)=\limsup _{t \rightarrow \infty} \frac{1}{t} \log r_{t}(F, \delta) .
$$

Notice that $h(\varphi \mid F, \delta)$ increases as $\delta$ decreases. Finally we let $h(\varphi \mid F)=$ $\lim _{\delta \rightarrow 0} h(\varphi \mid F, \delta)$. We denote $h(\varphi \mid X, \delta)$ and $h(\varphi \mid X)$ by $h(\varphi, \delta)$ and $h(\varphi)$ respectively.

In fact the topological entropy of a flow $(X, \varphi)$ equals the topological entropy of the homeomorphism $\varphi_{1}$, and more generally $h\left(\varphi_{t}\right)=|t| \cdot h\left(\varphi_{1}\right)$. For more details see [1].

Let $I$ be any interval of real numbers containing the origin. A reparametrization of $I$ is an orientation-preserving homeomorphism (increasing) from $I$ onto its image fixing the origin. Define $\operatorname{Rep}(I)$ and $\operatorname{Rep}(R)$ to be the sets of all reparametrizations of $I$ and $R$ respectively.

Lemma 1 (cf. [5, Lemma 2]). Let $(X, \varphi)$ be a flow with no fixed points. Then $\exists T_{0}>0$ such that if $0<t<T_{0}$, there is a $\gamma>0$ such that $d\left(\varphi_{t} x, x\right)>\gamma$ for any $x \in X$.

Lemma 2 (cf. [13, Lemma 1.2]): Let $(X, \varphi)$ be a flow with no fixed points. Then $\forall \lambda>0, \exists \varepsilon>0$ such that if $x, y \in X, I$ is a closed interval containing the origin, and $\alpha \in \operatorname{Rep}(I)$ with $d\left(\varphi_{\alpha(s)} x, \varphi_{s} y\right) \leq \varepsilon$ for all $s \in I$, then $|\alpha(s)-s| \leq \lambda$ for all $|s|<1$ in $I$ and $|\alpha(s)-s| \leq|s| \lambda$ for all $|s| \geq 1$ in $I$.

Let $(X, \varphi)$ be a flow and let $\varepsilon>0$. For $x \in X$ and $\gamma>\varepsilon$ define

$$
\begin{aligned}
& U(t, x, \gamma)=\left\{y \in X: d\left(\varphi_{\alpha(s)} y, \varphi_{s} x\right) \leq \gamma\right. \\
& \text { for some } \alpha \in \operatorname{Rep}(R) \text { and all } 0 \leq s \leq t\} .
\end{aligned}
$$

Let

$$
\bar{U}(t, x, \varepsilon)=\bigcap_{\gamma>\varepsilon} U(t, x, \gamma) .
$$

Proposition 3 (cf. [13, Proposition 1]). Let $(X, \varphi)$ be a flow without fixed points. Then for small $\varepsilon>0, \bar{U}(t, x, \varepsilon)$ is a closed subset of $X$ for every $x \in X$ and $t \geq 0$. 
Definition 4. A flow $(X, \varphi)$ is said to be strongly h-expansive if there is an $\varepsilon>0$ called the $h$-expansive constant, so that for every $x \in X$, the set $\xi_{\varepsilon}(x)=$ $\bigcap_{t \geq 0} \bar{U}(t, x, \varepsilon)$ has zero topological entropy (i.e., $h\left(\varphi, \xi_{\varepsilon}(x)\right)=0$ ).

Let $(X, \varphi)$ be any flow. For $E, F \subseteq X$ and $\delta>0$ we say that $E(t, \delta)$ weakly spans $F$ (with respect to $\varphi$ ), if for each $x \in F$, there is $e \in E$ and $\alpha \in \operatorname{Rep}(R)$ such that

$$
d\left(\varphi_{\alpha(s)} x, \varphi_{s} e\right) \leq \delta \text { for all } 0 \leq s \leq t .
$$

Let $R_{t}(F, \delta)=R_{t}(F, \delta, \varphi)$ denote the smallest cardinality of any $(t, \delta)$ weakly spanning set for $F$. Compactness of $F$ guarantees $R_{t}(F, \delta)<\infty$. Define

Notice that $H(\varphi \mid F, \delta)$ increases as $\delta$ decreases. Let

$$
H(\varphi \mid F, \delta)=\limsup _{t \rightarrow \infty} \frac{1}{t} \log R_{t}(F, \delta) .
$$

$$
H(\varphi \mid F)=\lim _{\delta \rightarrow 0} H(\varphi \mid F, \delta) .
$$

We denote $H(\varphi \mid X, \delta)$ and $H(\varphi \mid X)$ by $H(\varphi, \delta)$ and $H(\varphi)$ respectively [13].

Note that $H(\varphi) \leq h(\varphi)$.

Theorem 5 (cf. [13, Theorem A]). If $(X, \varphi)$ is a strongly h-expansive flow without fuxed points, then $H(\varphi)=h(\varphi)$.

Definition 6 [5]. We say that a flow $(X, \varphi)$ is expansive if $\forall \lambda>0, \exists \varepsilon>0$ with the property that if $d\left(\varphi_{s} x, \varphi_{\alpha(s)} y\right) \leq \varepsilon$ for all $s \in R$, for a pair of points $x, y \in X$, and a continuous map $\alpha: R \rightarrow R$ with $\alpha(0)=0$, then $y=\varphi_{s} x$, where $|s| \leq \lambda$.

This definition is clearly independent of the metric. Smales's Axiom A flows are also expansive when restricted to their nonwandering sets. For the definition of Axiom A flows and for more details see [2, 12].

Lemma 7 (cf. [5, Lemma 1]). If $(X, \varphi)$ is an expansive flow, then each fixed point is an isolated point of $X$.

This reduces the study of expansive flows to those without fixed points.

Proposition 8 (cf. [13, Proposition 4]). Every expansive flow $(X, \varphi)$ is strongly h-expansive.

\section{EXAMPLE}

In Proposition 3 we saw that $\bar{U}(t, x, \varepsilon)=\bigcap_{\gamma>\varepsilon} U(t, x, \gamma)$ is a closed subset of $X$. The following example shows that $U(t, x, \gamma)$ is not necessarily a closed subset of $X$.

Example 9. Consider two orbits $\varphi_{s}(x)$ and $\varphi_{s}(y)$ with $0 \leq s \leq t$, as illustrated in Figure (a). Let $N_{r}(p)$ denote the closed ball of radius $r$ and center $p$. Suppose $\bar{x}$ is a point in $\varphi_{[0, t]} x$ and $y_{1}, y_{2}$ and $x_{1}, x_{2}$ are points in the orbits 


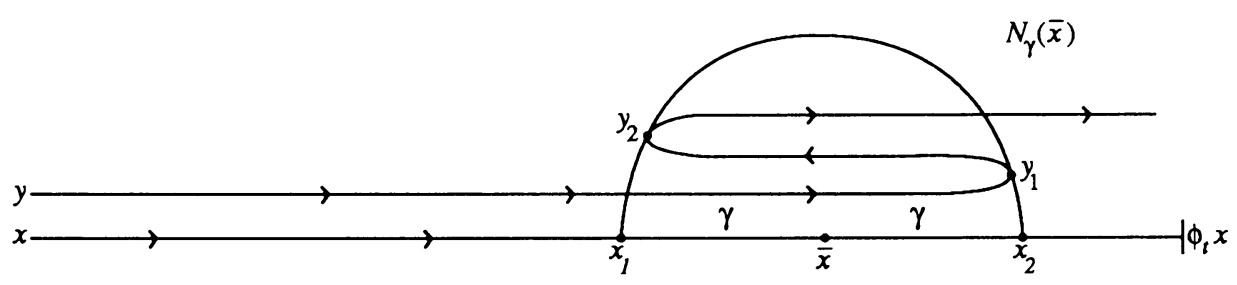

FIGURE (a)

of $y$ and $x$ respectively that lie in the boundary of $N_{\gamma}(\bar{x})$ for some $\gamma>0$ (as shown in Figure (a)). Assume $\alpha$ is any reparametrization of the orbit of $y$ and that $\varphi_{\alpha\left(s_{1}\right)} y=y_{1}$, for some $s_{1}$ with $0<s_{1}<t$. In order to keep $\varphi_{\alpha(s)}(y)$ and $\varphi_{s} x$ within distance $\gamma$ for all $0 \leq s \leq t, \varphi_{s_{1}} x$ must be to the right of $\bar{x}$ (as shown in Figure (a)). Suppose further that $\varphi_{\alpha\left(s_{2}\right)} y=y_{2}$ for some $s_{2}$ necessarily greater than $s_{1}$. Then $\varphi_{s_{2}} x$ is also to the right of $\bar{x}$. This means that $d\left(\varphi_{s_{2}} x, \varphi_{\alpha\left(s_{2}\right)} y\right)>\gamma$. Thus $y \notin U(t, x, \gamma)$.

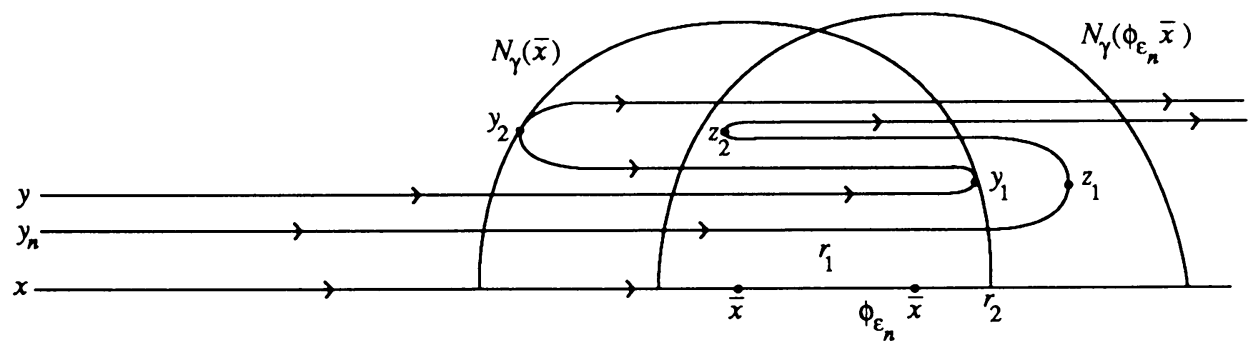

FIGURE (b)

We describe a sequence $\left\{y_{n}\right\}$ such that $\lim _{n \rightarrow \infty} y_{n}=y$ and $y_{n}$ is in $U(t, x, \gamma)$, for all $n$. This construction shows that $U(t, x, \gamma)$ is not closed. Figure (b) illustrates one of the sequences $\left\{y_{n}\right\}$ and its orbits. Let $\left\{\varepsilon_{n}\right\}$ be a sequence of real numbers such that $\varepsilon_{n} \rightarrow 0$, respectively. Define the orbit of $y_{n}$ so that the points of $z_{1}$ and $z_{2}$ are within the interior of $N_{\gamma}\left(\varphi_{\varepsilon_{n}} \bar{x}\right)$ (as shown in Figure (b)). Choose parameter values $s_{1}, s_{2}>0$ with $s_{1}<s_{2}$ so that $\varphi_{s_{1}} x=r_{1}$ is between $\bar{x}$ and $\varphi_{\varepsilon_{n}} \bar{x}, \varphi_{s_{2}} x=r_{2}$ is to the right of $\varphi_{\varepsilon_{n}} \bar{x}$, and at the same time $d\left(r_{1}, z_{1}\right)<\gamma$ and $d\left(r_{2}, z_{2}\right)<\gamma$. Then a reparametrization $\alpha$ can be assigned so that

$$
d\left(\varphi_{s} x, \varphi_{\alpha(s)} y\right)<\gamma \text { for all } 0 \leq s \leq t .
$$

Thus $y_{n} \in U(t, x, \gamma)$ for all $n$.

\section{TOPOLOGICAL ENTROPY}

In this section we will prove our conjecture raised in [13]. 
Theorem 10. If $(X, \varphi)$ is a continuous real flow without fxed points, then $H(\varphi)=h(\varphi)$.

In [13] we investigated topological entropies of mutually conjugate expansive flows. As an application of Theorem 10 one can generalize these results to any mutually conjugate flows without fixed points but free of expansiveness. See Theorem B, Corollary 1, and Corollary 2 in [13].

Let $(X, \varphi)$ be a flow. First we define the following:

For $E, F \subseteq X$ and $\varepsilon>0$ we say that $E(t, \varepsilon)$-traces $F$ (with respect to $\varphi$ ) if for each $x \in F$, there exist $e \in E$ and $\alpha \in \operatorname{Rep}(R)$ such that

$$
d\left(\varphi_{s} x, \varphi_{\alpha(s)} e\right) \leq \varepsilon \text { for all } 0 \leq s \leq t .
$$

Let $T_{t}(F, \varepsilon)$ denote the minimum cardinality of a set which $(t, \varepsilon)$-traces $F$. We define

$$
T(\varphi \mid F, \varepsilon)=\limsup _{t \rightarrow \infty} \frac{1}{t} \log T_{t}(F, \varepsilon) .
$$

The following lemma shows that this limit exists. Notice that $T(\varphi \mid F, \varepsilon)$ increases as $\varepsilon$ decreases. Let $T(\varphi \mid F)=\lim _{\varepsilon \rightarrow 0} T(\varphi \mid F, \varepsilon)$. We denote $T(\varphi \mid X, \varepsilon)$ by $T(\varphi, \varepsilon)$ and $T(\varphi \mid X)$ by $T(\varphi)$. Note that $T(\varphi) \leq h(\varphi)$. The following lemma shows that this definition $T(\varphi)$ is equivalent to $H(\varphi)$. From now on it is easier to deal with the definition of $T(\varphi)$ rather than $H(\varphi)$.

Lemma 11. Let $(X, \varphi)$ be a flow without fixed points.

(i) For all $\lambda>0$, there exists $\varepsilon>0$ such that $T_{(1-\lambda) t}(X, \delta) \leq R_{t}(X, \delta)$ and $R_{(1-\lambda) t}(X, \delta) \leq T_{t}(X, \delta)$ hold for all small enough $\delta, 0<\delta \leq \varepsilon$.

(ii) For $\varepsilon_{1}<\varepsilon_{2}, T\left(\varphi, \varepsilon_{2}\right) \leq T\left(\varphi, \varepsilon_{1}\right)$.

Proof. Take $\varepsilon>0$ satisfying Lemma 2. Given $0<\delta<\varepsilon$, and suppose $E$ is a $(t, \delta)$-weakly spanning set of $X$. Thus for every $x \in X$, there exist $e \in E$ and $\alpha \in \operatorname{Rep}(R)$ such that

$$
d\left(\varphi_{\alpha(s)} x, \varphi_{s} e\right) \leq \delta \text { for all } 0 \leq s \leq t .
$$

Take $u=\alpha(s)$. Thus we have

$$
d\left(\varphi_{u} x, \varphi_{\alpha^{-1}(u)} e\right) \leq \delta \text { for all } 0 \leq \alpha(s) \leq \alpha(t) .
$$

Lemma 2 implies that

$$
d\left(\varphi_{u} x, \varphi_{\alpha^{-1}(u)} e\right) \leq \delta \quad \text { for all } 0 \leq u \leq(1-\lambda) t .
$$

This means that $E$ is a $((1-\lambda) t, \delta)$-tracing set of $X$. Hence $T_{(1-\lambda) t}(X, \delta) \leq$ $R_{t}(X, \delta)$. Similarly we show that $R_{(1-\lambda) t}(X, \delta) \leq T_{t}(X, \delta)$. This finishes (i). (ii) is obvious.

The following is a direct consequence of Lemma 11.

Lemma 12. Let $(X, \varphi)$ be a flow without fixed points. Then $\forall \lambda>0, \exists \varepsilon>0$ such that

(i) $(1-\lambda) T(\varphi, \varepsilon) \leq H(\varphi, \varepsilon)$ and $(1-\lambda) H(\varphi, \varepsilon) \leq T(\varphi, \varepsilon)$, 
(ii) $(1-\lambda) T_{*}(\varphi, \varepsilon) \leq H_{*}(\varphi, \varepsilon)$ and $(1-\lambda) H_{*}(\varphi, \varepsilon) \leq T_{*}(\varphi, \varepsilon)$, where

$$
T_{*}(\varphi, \varepsilon)=\liminf _{t \rightarrow \infty} \frac{1}{t} \log T_{t}(X, \varepsilon),
$$

and

$$
H_{*}(\varphi, \varepsilon)=\liminf _{t \rightarrow \infty} \frac{1}{t} \log R_{t}(X, \varepsilon) .
$$

Corollary 13. If $(X, \varphi)$ is a flow without fixed points, then $H(\varphi)=T(\varphi)$ and $H_{*}(\varphi)=T_{*}(\varphi)$, where $H_{*}(\varphi)=\lim _{\varepsilon \rightarrow 0} H_{*}(\varphi, \varepsilon)$ and $T_{*}(\varphi)=\lim _{\varepsilon \rightarrow 0} T_{*}(\varphi, \varepsilon)$.

This corollary together with the following proposition finish the proof of Theorem 10.

Proposition 14. If $(X, \varphi)$ is a real flow without fuxed points, then $h(\varphi)=T(\varphi)$. Proof. Fix $e \in X$. Given an interval $I$ containing 0 and $\delta>0$ and $0<\varepsilon<$ $\delta / 3$, we shall say that $\alpha \in \operatorname{Rep}(I)$ is $\varepsilon$-good if there is $x \in X$ with

$$
d\left(\varphi_{s} x, \varphi_{\alpha(s)} e\right) \leq \varepsilon \quad \text { for all } s \in I .
$$

Choose $\theta>0$ so that $d\left(\varphi_{s} z, z\right) \leq \delta / 3$ whenever $z \in X$ and $|s| \leq \theta$. Given $\tau>0$, it is clear from Lemma 2 that we can choose $\varepsilon>0$ small enough so that if $I$ is any interval containing 0 and $\alpha \in \operatorname{Rep}(I)$ is $\varepsilon$-good, then

$$
\left|\left\{\alpha\left(s_{1}\right)-s_{1}\right\}-\left\{\alpha\left(s_{2}\right)-s_{2}\right\}\right| \leq \theta / 8 \tau,
$$

whenever $s_{1}, s_{2} \in I$ and $\left|s_{1}-s_{2}\right| \leq 1$ and so

$$
\left|\left\{\alpha\left(s_{1}\right)-s_{1}\right\}-\left\{\alpha\left(s_{2}\right)-s_{2}\right\}\right| \leq \theta / 4,
$$

whenever $s_{1}, s_{2} \in I$ and $\left|s_{1}-s_{2}\right| \leq \tau$. Associate to each $\varepsilon$-good $\alpha \in \operatorname{Rep}([0, t])$ the integer sequence

$$
\left\{\left[\frac{\alpha(k \tau)-k \tau}{\theta / 4}\right]\right\}, \quad k=0,1, \ldots,[t / \tau] .
$$

Suppose that $\alpha$ and $\beta$ are $\varepsilon$-good reparametrizations of $[0, t]$ with the same associated sequence. Then for any $s \in[0, t]$,

$$
\begin{aligned}
|\alpha(s)-\beta(s)| & =|\{\alpha(s)-s\}-\{\beta(s)-s\}| \\
& \leq|\{\alpha([s / \tau] \tau)-[s / \tau] \tau\}-\{\beta([s / \tau] \tau)-[s / \tau] \tau\}|+\theta / 4+\theta / 4 \\
& \leq \theta / 2+\theta / 2=\theta,
\end{aligned}
$$

and so $d\left(\varphi_{\alpha(s)} e, \varphi_{\beta(s)} e\right) \leq \delta / 3$ for $0 \leq s \leq t$.

Now suppose that $x, y \in X$ and there are $\alpha, \beta \in \operatorname{Rep}([0, t])$ such that $d\left(\varphi_{s} x, \varphi_{\alpha(s)} e\right)<\varepsilon$ and $d\left(\varphi_{s} y, \varphi_{\alpha(s)} e\right)<\varepsilon$ for $0<s<t$ and $\alpha$ and $\beta$ have the same associated sequence. Then for $0<s<t$,

$$
\begin{aligned}
d\left(\varphi_{s} x, \varphi_{s} y\right) & \leq d\left(\varphi_{s} x, \varphi_{\alpha(s)} e\right)+d\left(\varphi_{\alpha(s)} e, \varphi_{\beta(s)} e\right)+d\left(\varphi_{\beta(s)} e, \varphi_{s} y\right) \\
& \leq \varepsilon+\delta / 3+\varepsilon<\delta .
\end{aligned}
$$

For each integer sequence that can be associated with an $\varepsilon$-good $\alpha \in \operatorname{Rep}([0, t])$ choose one point $x \in X$ with

$$
d\left(\varphi_{s} x, \varphi_{\alpha(s)} e\right) \leq \varepsilon, \quad 0 \leq s \leq t
$$


It is clear from the above that the set of chosen points $(t, \delta)$-spans the set of points $(t, \varepsilon)$-traced by $\{e\}$. Observe that the integer sequence associated with an $\varepsilon$-good reparametrization has the property that consecutive terms differ by at most 1 and that the initial term is 0 . Hence there are at most

$$
3^{[t / \tau]+1} \leq 3.3^{t / \tau}
$$

such sequences. This means that the set of points $(t, \varepsilon)$-traced by $\{e\}$ is $(t, \delta)$ spanned by a set with at most $3.3^{t / \tau}$ elements. Thus one can begin with a $(t, \varepsilon)$-tracing set of cardinality $T_{t}(X, \varepsilon)$, replace each element by at most $3.3^{t / \tau}$ elements and obtain a $(t, \delta)$-spanning set $W$ with

$$
\text { card } W \leq 3.3^{t / \tau} T_{t}(X, \varepsilon) \text {. }
$$

Hence

and so

$$
\frac{1}{t} \log r_{t}(X, \varepsilon) \leq \frac{1}{t} \log 3+\frac{1}{\tau} \log 3+\frac{1}{t} \log T_{t}(X, \delta)
$$

$$
h(\varphi, \delta) \leq \frac{1}{\tau} \log 3+T(\varphi, \varepsilon) \leq \frac{1}{\tau} \log 3+T(\varphi) .
$$

This is true for arbitrarily large $\tau$ and arbitrarily small $\delta$. Thus $h(\varphi) \leq T(\varphi)$. Since $h(\varphi) \geq T(\varphi)$ is obvious, one has $h(\varphi)=T(\varphi)$ and the proof is finished.

\section{EXPANSIVE FLOWS}

Let $(X, \varphi)$ be a strongly $h$-expansive flow without fixed points. Then by Theorem 5 and Corollary 13 we have

$$
h(\varphi)=\lim _{\varepsilon \rightarrow 0} \limsup _{t \rightarrow \infty} \frac{1}{t} \log T_{t}(X, \varepsilon) .
$$

In [3] Bowen has shown that if $(X, \varphi)$ is an expansive flow, then for small $\varepsilon>0$ we have

$$
\frac{1}{t} \log r_{t}(X, \varepsilon) \rightarrow h(\varphi)
$$

as $t \rightarrow \infty$. In Proposition 14 we showed that $h(\varphi)=T(\varphi)$, but it is not yet known whether $(1 / t) \log T_{t}(X, \varepsilon)$ converges to $h(\varphi)$ for small $\varepsilon>0$. Theorem 22 is a result in this direction.

Lemma 15. $(X, \varphi)$ is an expansive flow if and only if $\forall \lambda>0, \exists \varepsilon>0$ with the property that $\forall \varepsilon_{0}>0, \exists T>0$ such that for every $x, y \in X$ and $\alpha \in \operatorname{Rep}(R)$ if $d\left(\varphi_{\alpha(t)} y, \varphi_{t} x\right) \leq \varepsilon$ for all $|t| \leq T$, then $d\left(\varphi_{r} y, x\right) \leq \varepsilon_{0}$ for some $r \in[-\lambda, \lambda]$.

Proof. Splice together Lemma 9 in [14] and Theorem 3(ii) in [5].

Lemma 16. Let $(X, \varphi)$ be a flow without fixed points. Then $\exists T_{0}>0$ such that if $0<T_{1} \leq T_{2}<T_{0}$, then $\exists \gamma>0$ with $d\left(\varphi_{u} x, x\right)>\gamma$ for all $u \in\left[T_{1}, T_{2}\right]$ and all $x \in X$.

Proof. Let $T_{0}$ satisfy Lemma 1 . Define $h:\left[T_{1}, T_{2}\right] \times X \rightarrow R$ by $h(u, x)=$ $d\left(\varphi_{u} x, x\right)>0$. Continuity of $h$ and compactness of $\left[T_{1}, T_{2}\right] \times X$ finish the proof. 
Lemma 17. Let $(X, \varphi)$ be an expansive flow. Then $\forall \lambda>0, \exists \varepsilon>0$ with the property that $\forall \varepsilon_{0}>0, \exists T>0$ such that if $x, y \in X, \alpha \in \operatorname{Rep}(R)$, and $t>0$ with

$$
d\left(\varphi_{\alpha(s)} y, \varphi_{s} x\right) \leq \varepsilon \text { for all }-T \leq s \leq T+t,
$$

then there exist $y^{\prime} \in \varphi_{[-\lambda, \lambda]} y$ and a continuous map $\beta:[0, t] \rightarrow R$ with $\beta(0)=$ 0 such that

$$
d\left(\varphi_{\beta(s)} y^{\prime}, \varphi_{s} x\right) \leq \varepsilon_{0} \quad \text { for all } 0 \leq s \leq t .
$$

Proof. Given $\lambda>0$, with $3 \lambda<T_{0}$ take $\varepsilon>0$ satisfying Lemma 15. Also given $\varepsilon_{0}>0$, take $\xi>0$ with $\xi<\lambda$ and satisfying the property that $d\left(x, \varphi_{r} x\right)<$ $\varepsilon_{0} / 3$ for all $0 \leq r \leq \xi$. Using Lemma 16 we can choose $\varepsilon_{0}^{\prime}>0$ such that $\varepsilon_{0}^{\prime}<\varepsilon_{0} / 3$ and $d\left(x, \varphi_{u} x\right)>\varepsilon_{0}^{\prime} / 3$ for all $u \in[\xi, 3 \lambda]$. Let $T>0$ satisfy Lemma 15 with respect to $\varepsilon_{0}^{\prime} / 3$. Now take $\gamma>0$ with the property that

(a) $0<\gamma<\xi$,

(b) $d\left(x, \varphi_{u} x\right) \leq \varepsilon_{0}^{\prime} / 3$ for all $0 \leq u \leq \gamma$,

(c) $|\alpha(t)-\alpha(s)| \leq \lambda$ provided that $|t-s| \leq \gamma$ (continuity of $\alpha$ ).

Consider a sequence $\left\{t_{i}\right\}_{i=0}^{m}$ with $t_{0}=0,0<t_{i+1}-t_{i}<\gamma$, and $t_{m}=t$. Now using Lemma 15 we can find a point $y^{\prime} \in \varphi_{[-\lambda, \lambda]} y$ and a sequence $\left\{u_{i}\right\}_{i=0}^{m}$ with $u_{0}=0, d\left(\varphi_{t_{i}} x, \varphi_{u_{i}} y^{\prime}\right) \leq \varepsilon_{0}^{\prime} / 3$ for all $i=0,1,2, \ldots, m$, and $\left|u_{i}-\alpha\left(t_{i}\right)\right| \leq \lambda$ for all $i=1,2,3, \ldots, m$. Thus,

$$
\begin{aligned}
d\left(\varphi_{u_{i}} y^{\prime}, \varphi_{u_{i-1}} y^{\prime}\right) & \leq d\left(\varphi_{u_{i}} y^{\prime}, \varphi_{t_{i}} x\right)+d\left(\varphi_{t_{i}} x, \varphi_{t_{i-1}} x\right)+d\left(\varphi_{t_{i-1}} x, \varphi_{u_{i-1}} y^{\prime}\right) \\
& \leq \varepsilon_{0}^{\prime} / 3+\varepsilon_{0}^{\prime} / 3+\varepsilon_{0}^{\prime} / 3=\varepsilon_{0}^{\prime} .
\end{aligned}
$$

Continuity of $\alpha$ and property (c) imply that

$$
\left|\alpha\left(t_{i}\right)-\alpha\left(t_{i-1}\right)\right| \leq \lambda \text { for all } i=1,2, \ldots, m \text {. }
$$

Hence

$$
\left|u_{i}-u_{i-1}\right| \leq\left|u_{i}-\alpha\left(t_{i}\right)\right|+\left|\alpha\left(t_{i}\right)-\alpha\left(t_{i-1}\right)\right|+\left|\alpha\left(t_{i-1}\right)-u_{i-1}\right| \leq 3 \lambda .
$$

This together with (i) and Lemma 16 force $\left|u_{i}-u_{i-1}\right|$ to be less than $\xi$. Now define $\beta:[0, t] \rightarrow R$ by $\beta\left(t_{i}\right)=u_{i}$ and by linearity on each interval $\left[t_{i}, t_{i+1}\right]$. Thus for $t_{i} \leq s \leq t_{i+1}$ we have

$$
\begin{aligned}
d\left(\varphi_{s} x, \varphi_{\beta(s)} y^{\prime}\right) & \leq d\left(\varphi_{s} x, \varphi_{t_{i}} x\right)+d\left(\varphi_{t_{i}} x, \varphi_{u_{i}} y^{\prime}\right)+d\left(\varphi_{u_{i}} y^{\prime}, \varphi_{\beta(s)} y^{\prime}\right) \\
& \leq \varepsilon_{0}^{\prime} / 3+\varepsilon_{0}^{\prime} / 3+\varepsilon_{0} / 3=\varepsilon_{0} .
\end{aligned}
$$

This finishes the proof.

The proof of the following lemma is contained in the proof of Theorem 3 in [5].

Lemma 18. Let $(X, \varphi)$ be a flow without fixed points. Then $\forall \xi$ with $0<\xi<$ $T_{0} / 3, \exists \varepsilon_{0}>0$ and $\exists \tau>0$ such that for every $x, y \in X$ and every continuous 
$\alpha: R \rightarrow R$ with $\alpha(0)=0$ if $d\left(\varphi_{s} x, \varphi_{\alpha(s)} y\right)<\varepsilon_{0}$ for all $0 \leq s \leq t$, then $\alpha(s+\xi)-\alpha(s) \geq \tau$ for all $0 \leq s \leq t$.

Lemma 19. Let $(X, \varphi)$ be an expansive flow. Then $\forall \lambda>0, \exists \varepsilon>0$ with the property that $\forall \varepsilon_{0}>0, \exists T>0$ such that if $x, y \in X, \alpha \in \operatorname{Rep}(R)$, and $t>0$ with $d\left(\varphi_{\alpha(s)} y, \varphi_{s} x\right) \leq \varepsilon$ for all $-T \leq s \leq T+t$, then there exist $y^{\prime} \in \varphi_{[-\lambda, \lambda]} y$ and $h \in \operatorname{Rep}(R)$ such that

$$
d\left(\varphi_{h(s)} y^{\prime}, \varphi_{s} x\right) \leq \varepsilon_{0} \quad \text { for all } 0 \leq s \leq t .
$$

Proof. Given $\lambda>0$, choose $\varepsilon>0$ satisfying Lemma 17. Now for any $\varepsilon_{0}>0$ we can choose $n$ sufficiently large so that $0<t / n \leq T_{0} / 3$ and $d\left(x, \varphi_{u} x\right)<$ $\varepsilon_{0} / 2$ for all $0 \leq u \leq t / n$ and for all $x \in X$. Choose $\varepsilon_{0}^{\prime}$ with $0<\varepsilon_{0}^{\prime}<\varepsilon_{0} / 2$ and $\tau>0$ satisfying Lemma 18 with respect to $t / n$ (i.e., $\alpha(t / n+s)-\alpha(s) \geq \tau)$. Also let $T>0$ satisfy Lemma 17 with respect to $\varepsilon_{0}^{\prime}$. Now assume

$$
d\left(\varphi_{s} x, \varphi_{\alpha(s)} y\right) \leq \varepsilon \text { for all }-T \leq s \leq T+t
$$

for some $x, y \in X$ and $\alpha \in \operatorname{Rep}(R)$. Thus by Lemma 17 there exist $y^{\prime} \in$ $\varphi_{[-\lambda, \lambda]} y$ and a continuous $\beta[0, t] \rightarrow R$ with $\beta(0)=0$ such that

$$
d\left(\varphi_{s} x, \varphi_{\beta(s)} y^{\prime}\right) \leq \varepsilon_{0}^{\prime} \text { for all } 0 \leq s \leq t .
$$

Now using Lemma 18 we have $\beta(s+t / n)-\beta(s) \geq \tau$ for all $0 \leq s \leq$ $(n+1) t / n$. Define $h:[0, t] \rightarrow R$ by $h(i t / n)=\beta(i t / n)$ for all $i=0,1,2, \ldots$, and by linearity on each $[i t / n,(i+1) t / n]$. It is obvious that $h$ is an increasing homeomorphism from $[0, t]$ onto its image and can be extended to $h \in \operatorname{Rep}(R)$. Now for $s \in[i t / n,(i+1) t / n]$, there exists $s^{\prime} \in[i t / n,(i+1) t / n]$ with $h(s)=\beta\left(s^{\prime}\right)$. Hence

$$
\begin{aligned}
d\left(\varphi_{s} x, \varphi_{h(s)} y^{\prime}\right) & =d\left(\varphi_{s} x, \varphi_{\beta\left(s^{\prime}\right)} y^{\prime}\right) \leq d\left(\varphi_{s} x, \varphi_{s^{\prime}} x\right)+d\left(\varphi_{s^{\prime}}, x, \varphi_{\beta\left(s^{\prime}\right)} y^{\prime}\right) \\
& \leq d\left(\varphi_{s-s^{\prime}} \varphi_{s^{\prime}} x, \varphi_{s^{\prime}} x\right)+d\left(\varphi_{s^{\prime}} x, \varphi_{\beta\left(s^{\prime}\right)} y^{\prime}\right) \leq \varepsilon_{0} / 2+\varepsilon_{0}^{\prime} \leq \varepsilon_{0} .
\end{aligned}
$$

This finishes the proof.

Proposition 20. Let $(X, \varphi)$ be an expansive flow. Then $\exists \varepsilon>0$ such that $T(\varphi)=T(\varphi, \varepsilon)$ and $T_{*}(\varphi)=T(\varphi, \varepsilon)$.

Proof. Given $\lambda>0$, choose $\varepsilon>0$ satisfying Lemma 19 with respect to $\lambda$. For any $\varepsilon_{0}>0$, there exists $T>0$ also satisfying Lemma 19. Suppose $E$ is a $(2 T+t, \varepsilon)$-tracing set of $X$ (i.e., $\forall x \in X, \exists y \in E$ and $\exists \alpha \in \operatorname{Rep}(R)$ such that $d\left(\varphi_{\alpha(s)} y, \varphi_{s} x\right) \leq \varepsilon$ for all $\left.0 \leq s \leq 2 T+t\right)$. Take $u=s-T$ and therefore

$$
d\left(\varphi_{\alpha(u+T)} y, \varphi_{u+T} x\right) \leq \varepsilon \text { for all }-T \leq u \leq T+t .
$$

Hence

$$
d\left(\varphi_{\alpha(u+T)-\alpha(T)} \varphi_{\alpha(T)} y, \varphi_{u} \varphi_{T} x\right) \leq \varepsilon \text { for all }-T \leq u \leq T+t .
$$

Let $\beta(u)=\alpha(u+T)-\alpha(T)$. Then $\beta \in \operatorname{Rep}(R)$ and

$$
d\left(\varphi_{\beta(u)} \varphi_{\alpha(T)} y, \varphi_{u} \varphi_{T} x\right) \leq \varepsilon \text { for all }-T \leq u \leq T+t .
$$


Using Lemma 19, there exists $y^{\prime} \in \varphi_{[-\lambda, \lambda]} \varphi_{\alpha(T)} y$ and $h \in \operatorname{Rep}(R)$ so that

$$
d\left(\varphi_{h(s)} y^{\prime}, \varphi_{s} \varphi_{T} x\right) \leq \varepsilon_{0} \text { for all } 0 \leq s \leq t .
$$

Now applying Lemma 2 we have $(1-\lambda) T \leq \alpha(T) \leq(1+\lambda) T$. Thus $y^{\prime}=\varphi_{v} y$ with $v \in[(1-\lambda) T-\lambda,(1+\lambda) T+\lambda]$. Fix a sufficiently large integer $n$ such that if $\delta=2 \lambda(T+1) / n$, then $d\left(\varphi_{\delta} x, x\right) \leq \varepsilon_{0}$ for all $x \in X$. Now for $y \in E$, take $z=\varphi_{(1-\lambda) T-\lambda} y$ and define

$$
E_{y}=\left\{z, \varphi_{\delta} z, \delta_{2 \delta} z, \ldots, \varphi_{n \delta} z\right\} .
$$

It is obvious that $E_{y}$ is a $\left(t, \varepsilon_{0}\right)$-spanning set of

$$
\left\{\varphi_{v} y: v \in[(1-\lambda) T-\lambda,(1+\lambda) T+\lambda]\right\}
$$

whose cardinality $\leq n+1$. Let $W=\bigcup_{y \in E} E_{y}$. Then

$$
\operatorname{card}(W) \leq(n+1) \operatorname{card}(E) \text {. }
$$

To show that $W$ is a $\left(t, 2 \varepsilon_{0}\right)$-tracing set of $X$, let $x \in X$ and take $\varphi_{-T} x$. Then since $E$ is a $(2 T+t, \varepsilon)$-tracing set of $X$, there exist $y \in E$ and $\alpha \in \operatorname{Rep}(R)$ so that

$$
d\left(\varphi_{\alpha(s)} y, \varphi_{s} \varphi_{-T} x\right) \leq \varepsilon \text { for all } 0 \leq s \leq 2 T+t .
$$

By taking $u=s-T$ we have

$$
d\left(\varphi_{\alpha(u+t)} y, \varphi_{u} x\right) \leq \varepsilon \text { for all }-T \leq s \leq T+t .
$$

Hence

$$
d\left(\varphi_{\alpha(u+t)-\alpha(T)} \varphi_{\alpha(T)} y, \varphi_{u} x\right) \leq \varepsilon \text { for all }-T \leq s \leq T+t .
$$

Lemma 19 implies that there exist $y^{\prime} \in \varphi_{[-\lambda, \lambda]} \varphi_{\alpha(T)} y$ and $h \in \operatorname{Rep}(R)$ so that

$$
d\left(\varphi_{h(u)} y^{\prime}, \varphi_{u} x\right) \leq \varepsilon_{0} \text { for all } 0 \leq u \leq t .
$$

Since

$$
y^{\prime} \in\left\{\varphi_{v} y: v \in[(1-\lambda) T-\lambda,(1+\lambda) T+\lambda]\right\}
$$

by Lemma 2, it follows that there exists $w \in E_{y}$ with

$$
d\left(\varphi_{h(u)} y^{\prime}, \varphi_{h(u)} w\right) \leq \varepsilon_{0} \quad \text { for all } u \in R .
$$

The triangle inequality implies that

$$
d\left(\varphi_{h(u)} w, \varphi_{u} x\right) \leq 2 \varepsilon_{0} \text { for all } 0 \leq u \leq t .
$$

This shows that $W$ is a $\left(t, 2 \varepsilon_{0}\right)$-tracing set of $X$. This means that $T\left(\varphi, 2 \varepsilon_{0}\right) \leq$ $T(\varphi, \varepsilon)$ and $T_{*}\left(\varphi, 2 \varepsilon_{0}\right) \leq T_{*}(\varphi, \varepsilon)$ for any $\varepsilon_{0}>0$. Thus $T(\varphi)=T(\varphi, \varepsilon)$ and $T_{*}(\varphi)=T_{*}(\varphi, \varepsilon)$, and the proof is finished.

Proposition 21. If $(X, \varphi)$ is an expansive flow without fixed points, then $T(\varphi)=$ $T_{*}(\varphi)$.

Proof. It is easy to adapt the proof of Proposition 5 and Theorem A in [13] to show that

$$
(1-\lambda) h(\varphi) \leq \liminf _{s \rightarrow \infty} \frac{1}{s} \log \operatorname{card}(E)=H_{*}(\varphi, \varepsilon) .
$$


Lemma 12 implies that $(1-\lambda) H_{*}(\varphi, \varepsilon) \leq T_{*}(\varphi, \varepsilon)$. Thus, $(1-\lambda)^{2} h(\varphi) \leq$ $T_{*}(\varphi, \varepsilon)$. But $T_{*}(\varphi, \varepsilon) \leq T(\varphi, \varepsilon) \leq T(\varphi) \leq h(\varphi)$. Therefore,

$$
T(\varphi, \varepsilon)-T_{*}(\varphi, \varepsilon) \leq 2 \lambda h(\varphi)-\lambda^{2} h(\varphi) \leq \gamma .
$$

As $\gamma \rightarrow 0$ we have $T(\varphi)=T_{*}(\varphi)$.

Combining this proposition with Corollary 13 and Proposition 20, we have Theorem 22. If $(X, \varphi)$ is an expansive flow, then $\exists \varepsilon>0$ such that

$$
h(\varphi)=\lim _{t \rightarrow \infty} \frac{1}{t} \log T_{t}(X, \varepsilon) .
$$

Note that this theorem sharpens Bowen's result from [3].

\section{Global ENTROPY}

In this section we would like to answer an important question whether the topological entropy can be defined globally on local cross sections (i.e., whether the present can tell us about the past and the future).

Let $(X, \varphi)$ be a flow without fixed points. $S \subseteq X$ is called a local cross section of time $\xi>0$ if $S$ is a closed subset of $X$ and $S \cap \varphi_{[-\xi, \xi]} x=\{x\}$ for all $x \in S$. If $S$ is a cross section of time $\xi$, then $\varphi_{[-\xi, \xi]} S$ and $S \times[-\xi, \xi]$ are homemorphic. By the interior $S^{*}$ of $S$ we mean the set $S \cap \operatorname{int}\left(\varphi_{[-\xi, \xi]} S\right)$. Note that $\varphi_{(-\varepsilon, \varepsilon)} S^{*}$ is open in $X$ for every $0<\varepsilon \leq \xi$. A theorem of Whitney [16], asserts that for each $x \in X$ there is a local cross section $S_{x}$ of time $v_{x}$ with $x \in S_{x}^{*}$. For more details see also [5].

Lemma 23 (cf. [5, Lemma 7]). Let $(X, \varphi)$ be a flow without fixed points. Then there is $a \xi>0$ such that for each $\alpha>0$ we can find a finite family $\mathbf{S}=\left\{S_{1}, S_{2}, \ldots, S_{m}\right\}$ of pairwise local cross sections of time $\boldsymbol{\xi}$ and diameter at most $\alpha$ so that

$$
X=\varphi_{[-\alpha, 0]} S^{+}=\varphi_{[0, \alpha]} S^{+}, \quad \text { where } S^{+}=\bigcup_{i=1}^{m} S_{i} .
$$

Take $\alpha<\xi / 3$ in the above lemma and let $\beta$ be the minimum time distance between sections of $\mathbf{S}$, i.e.,

$$
\beta=\sup \left\{\delta>0: \forall x \in S^{+} \text {we have } \varphi_{(0, \delta)} x \cap S^{+}=\varnothing\right\} .
$$

Note that $0<\beta \leq \alpha$. Take $p>0$ with $0<p<\beta / 2, p<T_{0} / 2$, and $p<\xi / 2$. For $S \in \mathbf{S}$ let $D_{p}=\varphi_{[-p, p]} S$ and define a projection map $\pi_{p}: D_{p} \rightarrow S$ by $\pi_{p}(x)=\varphi_{t}(x)$ where $\varphi_{t}(x) \in S$ and $|t| \leq p$. Since $2 p<\xi$, it follows that $\pi_{p}$ is well defined, continuous, and onto $S$ [10]. Keynes and Sears in [10] used the idea of local cross section and have shown that a flow supporting expansiveness must be finite dimensional.

From now on and as a standing hypothesis we assume that every flow $(X, \varphi)$ with no fixed points has a fixed family $\mathbf{S}$ of local cross section satisfying Lemma 23 and the above argument.

We would like to raise the following: 
Conjecture. If $(X, \varphi)$ is an expansive flow, then there is a metric $\rho$ compatible with the relative topology on $S^{+}$and there is $\lambda$ with $0<\lambda<1$ such that

$$
h(\varphi)=\lim _{t \rightarrow \infty} \frac{1}{t} \log N_{\rho}\left(\lambda^{t}\right),
$$

where $N_{\rho}\left(\lambda^{t}\right)$ is the minimum cardinality of a $\lambda^{t}$-net of $X$.

Note that $N_{\rho}\left(\lambda^{t}\right)=r_{0}\left(S^{+}, \lambda^{t}\right)$ with respect to the metric $\rho$ on $S^{+}$.

In this section we will use an adaptation of work by Reddy [11] to prove this conjecture under certain additional assumptions.

The following lemma can be proved also by using a similar argument to that used in Lemma 19.

Lemma 24. Let $(X, \varphi)$ be an expansive flow. Then $\forall \lambda>0, \exists \varepsilon>0$ with the property that $\forall \varepsilon_{0}>0, \exists T>0$ such that if $x, y \in X, \alpha \in \operatorname{Rep}(R)$, and $t>0$ with

$$
d\left(\varphi_{\alpha(s)} y, \varphi_{s} x\right) \leq \varepsilon \text { for all }-T-t \leq s \leq T+t .
$$

Then there exist $y^{\prime} \in \varphi_{[-\lambda, \lambda]} y$ and $h \in \operatorname{Rep}(R)$ such that

$$
d\left(\varphi_{h(s)} y^{\prime}, \varphi_{s} x\right) \leq \varepsilon_{0} \quad \text { for all }-t \leq s \leq t .
$$

Lemma 25. Let $(X, \varphi)$ be an expansive flow. Then $\exists \varepsilon>0$ with the property that $\forall \varepsilon_{0}>0, \exists T>0$ such that if $x, y \in S^{+}, \alpha \in \operatorname{Rep}(R)$, and $t>0$ with

$$
d\left(\varphi_{\alpha(s)} y, \varphi_{s} x\right) \leq \varepsilon \text { for all }-T-t \leq s \leq T+t,
$$

then $\exists h \in \operatorname{Rep}(R)$ such that

$$
d\left(\varphi_{h(s)} y, \varphi_{s} x\right) \leq \varepsilon_{0} \text { for all }-t \leq s \leq t .
$$

Proof. Given $\lambda>0$ with $\lambda<p$, take $\varepsilon>0$ satisfying Lemma 24 with respect to $\lambda$ such that if $x, y \in S^{+}$with $d(x, y) \leq \varepsilon$, then $x, y \in S$ for some $S \in \mathbf{S}$. Given $\varepsilon_{0}>0$, take $r>0$, with $r<\lambda$ and $d\left(x, \varphi_{u} x\right) \leq \varepsilon_{0} / 2$ for all $|u| \leq r$ and every $x \in X$. By Lemma 16 take $\gamma>0$ with $\gamma<\varepsilon_{0} / 2$ so that $d\left(x, \varphi_{u} x\right) \leq \gamma$ for all $u \in[r, p]$. Choose $\gamma^{\prime}>0$ with $\gamma^{\prime}<\gamma / 2$ and satisfy the continuity of $\pi_{p}$ with respect to $\gamma / 2$. Also let $T$ satisfy Lemma 24 with respect to $\gamma^{\prime}$ and assume

$$
d\left(\varphi_{\alpha(s)} y, \varphi_{s} x\right) \leq \varepsilon \text { for all }-T-t \leq s \leq T+t .,
$$

Thus by Lemma 24, there exist $y^{\prime} \in \varphi_{[-\lambda, \lambda]} y$ and $h \in \operatorname{Rep}(R)$ such that

$$
d\left(\varphi_{h(s)} y^{\prime}, \varphi_{s} x\right) \leq \gamma^{\prime} \quad \text { for all }-t \leq s \leq t .
$$

When $s=0, d\left(y^{\prime}, x\right) \leq \gamma^{\prime}$. Thus by continuity of $\pi_{p}$ we have $d\left(\pi_{p} y^{\prime}, \pi_{p} x\right) \leq$ $\gamma / 2$. But $\pi_{p} x=x$ and $\pi_{p} y^{\prime}=y$, therefore $d(y, x) \leq \gamma / 2$. The triangle inequality implies that $d\left(y, y^{\prime}\right) \leq \gamma$. This with Lemma 16 forces the time distances between $y$ and $y^{\prime}$ to be at most $r$. This means that

$$
d\left(\varphi_{h(s)} y, \varphi_{h(s)} y^{\prime}\right) \leq \varepsilon_{0} / 2 \text { for all } s \in R .
$$


Again using triangle inequality we have

$$
d\left(\varphi_{h(s)} y, \varphi_{s} x\right) \leq \varepsilon_{0} \text { for all }-t \leq s \leq t,
$$

and the proof is finished.

Let $(X, \varphi)$ be any flow. For $E, F \subseteq X$ and $\varepsilon>0$ we sat that $E(\overline{t, \varepsilon})$ traces $F$ (with respect to $\varphi$ ) if each $x \in F$, there exist $e \in E$ and $\alpha \in \operatorname{Rep}(R)$ such that

$$
d\left(\varphi_{\alpha(s)} e, \varphi_{s} x\right) \leq \varepsilon \text { for all }-t \leq s \leq t .
$$

Let $\bar{T}_{t}(X, \varepsilon)$ be the smallest cardinality of any $(\overline{t, \varepsilon})$-tracing set of $X$. Define

$$
\bar{T}(\varphi, \varepsilon)=\limsup _{t \rightarrow \infty} \frac{1}{t} \log \bar{T}_{t}(X, \varepsilon),
$$

and

$$
\bar{T}(\varphi)=\lim _{\varepsilon \rightarrow 0} \bar{T}(\varphi, \varepsilon) .
$$

The following lemma shows that these limits exist. We call $\bar{T}(\varphi)$ two-sided topological entropy.

Lemma 26. Let $(X, \varphi)$ be a flow without fixed points. Then $\bar{T}(\varphi)=2 T(\varphi)$.

Proof. Given $\lambda>0$, take $\varepsilon>0$ satisfying Lemma 2 with respect to $\lambda$. Let $E$ be any $(2 t, \varepsilon)$-tracing set of $X$. Thus for $\varphi_{-t} x \in X$, there exist $e \in E$ and $\alpha \in \operatorname{Rep}(R)$ such that

$$
d\left(\varphi_{\alpha(s)} e, \varphi_{s} \varphi_{-t} x\right) \leq \varepsilon \text { for all } 0 \leq s \leq 2 t .
$$

Let $u=s-t$. Then

$$
d\left(\varphi_{\alpha(u+t)-\alpha(t)} \varphi_{\alpha(t)} e, \varphi_{u} x\right) \leq \varepsilon \text { for all }-t \leq u \leq t .
$$

Thus,

$$
d\left(\varphi_{\beta(u)} \varphi_{\alpha(t)} e, \varphi_{u} x\right) \leq \varepsilon \text { for all }-t \leq u \leq t,
$$

where $\beta(u)=\alpha(u+t)-\alpha(t)$. By Lemma 2 we have $\alpha(t) \in[(1-\lambda) t,(1+\lambda) t]$. Fix $r>0$ sufficiently small such that $d\left(\varphi_{u} x, x\right) \leq \varepsilon$ for all $x \in X$ and for all $|u| \leq r$. Now for every $e \in E$ we consider the set

$$
E_{e}=\left\{\varphi_{(1-\lambda) t} e, \varphi_{(1-\lambda) t+r} e, \ldots, \varphi_{(1-\lambda) t+n r} e\right\},
$$

where $n$ is a positive integer with $2 \lambda t \leq n r \leq 2 \lambda t+1$. Take $W=\bigcup_{e \in E} E_{e}$. Thus $W$ is a $(\overline{t, 2 \varepsilon})$-tracing set of $X$ and

$$
\operatorname{card}(W) \leq(n+1) \operatorname{card}(E) .
$$

Hence

$$
\operatorname{card}(W) \leq((2 \lambda t+1) / r+1) \operatorname{card}(E),
$$

and

$$
\frac{1}{t} \log \operatorname{card}(W) \leq \frac{1}{t} \log ((2 \lambda t+1) / r+1)+\frac{1}{t} \log \operatorname{card}(E) .
$$

This means that $\bar{T}(\varphi, 2 \varepsilon) \leq 2 T(\varphi, \varepsilon)$. Using a similar argument we can show that $2 T(\varphi, 2 \varepsilon) \leq \bar{T}(\varphi, \varepsilon)$, and the proof is finished. 
Using the proof of the above lemma and Theorem 22 we can show

Corollary 27. If $(X, \varphi)$ is an expansive flow, the $\exists \varepsilon>0$ such that

$$
\bar{T}(\varphi)=\lim _{t \rightarrow \infty} \frac{1}{t} \log \bar{T}_{t}(X, \varepsilon) .
$$

Lemma 28. Let $(X, \varphi)$ be a flow without fixed points and let $F$ be any closed subset of $X$ with $\varphi_{[0, r]} F=X$ for some $r>0$. Then

(i) $h(\varphi \mid F)=h(\varphi)$,

(ii) $T(\varphi \mid F)=T(\varphi)$,

(ii) $\bar{T}(\varphi \mid F)=\bar{T}(\varphi)$,

(iv) $\bar{T}(\varphi \mid F)=2 T(\varphi \mid F)$.

Proof. Given $\lambda>0$, take $\varepsilon>0$ satisfying Lemma 2 with respect to $\lambda$. To prove (ii), let $E$ be any $(t, \varepsilon)$-tracing set of $F$. Fix a large positive integer $n$ such that $d\left(x, \varphi_{u} x\right) \leq \varepsilon$ for all $|u| \leq r(1+\lambda) / n$. For $e \in E$ define

$$
E_{e}=\left\{e, \varphi_{\delta} e, \varphi_{2 \delta} e, \ldots, \varphi_{n \delta} e\right\} \text {, }
$$

where $\delta=r(1+\lambda) / n$. It is obvious that for any $s>0$ we have $E_{e}$ is a $(s, \varepsilon)$ spanning set of the set $\varphi_{[0, r(1+\lambda)]} e$ and $\operatorname{card}\left(E_{e}\right) \leq n+1$. Take $W=\bigcup_{e \in E} E_{e}$. In order to prove that $W$ is a $(t-r, 2 \varepsilon)$-tracing set of $X$, let $x \in X$. Take $v>0$ with $0 \leq v \leq r$ and $\varphi_{-v} x \in F$. Thus there exist $e \in E$ and $\alpha \in \operatorname{Rep}(R)$ so that

$$
d\left(\varphi_{s} \varphi_{-v} x, \varphi_{\alpha(s)} e\right) \leq \varepsilon \quad \text { for all } 0 \leq s \leq t
$$

Let $u=s-v$. Then

$$
d\left(\varphi_{u} x, \varphi_{\alpha(u+v)} e\right) \leq \varepsilon \quad \text { for all } 0 \leq s \leq t-r .
$$

Thus,

$$
d\left(\varphi_{u} x, \varphi_{\beta(u)} \varphi_{\alpha(v)} e\right) \leq \varepsilon \text { for all } 0 \leq s \leq t-r,
$$

where $\beta(u)=\alpha(u+v)-\alpha(v)$. Using Lemma 2 we have $\alpha(v) \in[0,(1+\lambda) r]$. Therefore there exists $w \in E_{e}$ such that

$$
d\left(\varphi_{s} w, \varphi_{s} \varphi_{\alpha(v)} e\right) \leq \varepsilon \quad \text { for all } s \geq 0
$$

Thus by the triangle inequality we have

$$
d\left(\varphi_{\beta(s)} w, \varphi_{s} x\right) \leq 2 \varepsilon \quad \text { for all } 0 \leq s \leq t-r .
$$

This means that $W$ is a $(t-r, 2 \varepsilon)$-tracing set of $X$ with $\operatorname{card}(W) \leq$ $(n+1) \operatorname{card}(E)$. This implies that $T(\varphi) \leq T(\varphi \mid F)$. But we know that $T(\varphi \mid F) \leq$ $T(\varphi)$. Therefore $T(\varphi)=T(\varphi \mid F)$ and the proof of (ii) is finished.

The proof of (i) and (iii) is similar to the above and is an easy exercise for the reader.

Using Lemma 27 with (ii) and (iii) we have $\bar{T}(\varphi \mid F)=\bar{T}(\varphi)=2 T(\varphi)=$ $2 T(\varphi \mid F)$. This finishes (iv).

Definition 29. We say that a flow $(X, \varphi)$ is a uniformly expansive if $(X, \varphi)$ is expansive with the property that there are $r, \varepsilon>0$ such that if $x, y \in X$, $\left[T_{1}, T_{2}\right]$ is an interval containing zero, and $\alpha \in \operatorname{Rep}(R)$ with

$$
d\left(\varphi_{\alpha(s)} x, \varphi_{s} y\right) \leq \varepsilon \text { for all } T_{1} \leq s \leq T_{2},
$$

then $|\alpha(s)-s| \leq r$ for all $T_{1} \leq s \leq T_{2}$. 
Axiom $A$ flows satisfy Definition 29 because Lemma 3.2 in [7] and Proposition 1.6 in [2] show that they satisfy an even stronger property.

(5.1) Standing hypothesis. We shall assume throughout the remainder of this section that $(X, \varphi)$ is a uniformly expansive flow.

Lemma 7 reduces the study of expansive flows to those without fixed points.

Let $(X, \varphi)$ be a flow and fix $r>0$ satisfying Definition 29 .

Lemma 30. $\exists \varepsilon>0$ with the property that $\forall \varepsilon_{0}, r>0, \exists T>0$ such that if $x, y \in S^{+}$and $\varphi \in \operatorname{Rep}(R)$ and $t>0$ with

$$
d\left(\varphi_{\alpha(s)} y, \varphi_{s} x\right) \leq \varepsilon \text { for all }-T-t \leq s \leq T+t,
$$

then $\exists h \in \operatorname{Rep}(R)$ such that

$$
D\left(\varphi_{h(s)} y, \varphi_{s} x\right) \leq \varepsilon_{0} \text { for all }-t-2 r \leq s \leq t+2 r .
$$

Proof. Take $\varepsilon$ satisfying Lemma 25. Also given $\varepsilon_{0}>0$, take $\delta>0$ such that if $d(x, y) \leq \delta$, then $d\left(\varphi_{t} x, \varphi_{t} y\right) \leq \varepsilon_{0}$ for all $|t| \leq 2 r$. Thus there exists $T>0$ which satisfies Lemma 25 with respect to $\delta$, and the rest follows easily.

From now on fix $\varepsilon>0$ satisfying Lemma 30 and with the property that if $x, y \in S^{+}$and $d(x, y) \leq \varepsilon$, then $x, y \in S$ for some $S \in \mathbf{S}$.

For $t \geq 0$ define,

$$
W_{t}=\left\{(x, y) \in S^{+} \times S^{+}: d\left(\varphi_{\alpha(s)} x, \varphi_{s} y\right) \leq \varepsilon, d\left(\varphi_{\beta(s)} y, \varphi_{s} x\right) \leq \varepsilon\right.
$$

for some $\alpha, \beta \in \operatorname{Rep}(R)$ and for all $-t \leq s \leq t\}$.

Fix $\varepsilon_{0}>0$ small enough such that $3 \varepsilon_{0} \leq \varepsilon$. Also fix $T>0$ which satisfies Lemma 30 with respect to $\varepsilon_{0}$. Define $V_{0}=W_{0}=S^{+} \times S^{+}$and $V_{n}=W_{n T}$ for $n=1,2,3, \ldots$.

Lemma 31. The sequence $\left\{V_{n}\right\}$ is a nested sequence of symmetric neighborhoods of $\Delta=\left\{(x, x): x \in S^{+}\right\}$whose intersection is $\Delta$ such that $V_{n+1} \circ V_{n+1} \circ V_{n+1} \subseteq V_{n}$ for all $n \geq 0$.

Proof. Let $(x, y) \in V_{n}$ for all $n \geq 0$. Lemma 25 implies that for any arbitrary $\delta>0$, there exists a positive integer $n$ such that if $(x, y) \in V_{n}$, then $d(x, y) \leq$ $\delta$. This means that $x=y$. Thus, $\cap V_{n}=\Delta$. To prove $V_{n+1} \circ V_{n+1} \circ V_{n+1} \subseteq V_{n}$, let $(x, y) \in V_{n+1} \circ V_{n+1} \circ V_{n+1}$. There exist $a, b \in S^{+}$and $\alpha_{i}, \beta_{i} \in \operatorname{Rep}(R)$ for $i=1,2,3$ such that for all $s$ with $-(n+1) T \leq s \leq(n+1) T$ we have

$$
\begin{aligned}
& d\left(\varphi_{\alpha_{1}(s)} x, \varphi_{s} a\right) \leq \varepsilon \quad \text { and } \quad d\left(\varphi_{\beta_{1}(s)} a, \varphi_{s} x\right) \leq \varepsilon, \\
& d\left(\varphi_{\alpha_{2}(s)} a, \varphi_{s} b\right) \leq \varepsilon \quad \text { and } d\left(\varphi_{\beta_{2}(s)} b, \varphi_{s} a\right) \leq \varepsilon,
\end{aligned}
$$

and

$$
d\left(\varphi_{\alpha_{3}(s)} b, \varphi_{s} y\right) \leq \varepsilon \text { and } d\left(\varphi_{\beta_{3}(s)} y, \varphi_{s} b\right) \leq \varepsilon .
$$

Using Lemma 30, there exist $h_{i}, \gamma_{i} \in \operatorname{Rep}(R)$ for $i=1,2,3$ such that for all $s$ with $-n T-2 r \leq s \leq n T+2 r$ we have

(i) $d\left(\varphi_{h_{1}(s)} x, \varphi_{s} a\right) \leq \varepsilon_{0}$ and $d\left(\varphi_{\gamma_{1}(s)} a, \varphi_{s} x\right) \leq \varepsilon_{0}$, 
(ii) $d\left(\varphi_{h_{2}(s)} a, \varphi_{s} b\right) \leq \varepsilon_{0}$ and $d\left(\varphi_{\gamma_{2}(s)} b, \varphi_{s} a\right) \leq \varepsilon_{0}$, and

(iii) $d\left(\varphi_{h_{3}(s)} b, \varphi_{s} y\right) \leq \varepsilon_{0}$ and $d\left(\varphi_{\gamma_{3}(s)} y, \varphi_{s} b\right) \leq \varepsilon_{0}$.

By Definition 29 we have $\left|h_{i}(s)-s\right| \leq r$ and $\left|\beta_{i}(s)-s\right| \leq r$ for all $i=1,2,3$ and for all $-n T-2 r \leq s \leq n T+2 r$. Thus replacing $h_{2}(s)$ by $s$ in (i) we have

$$
d\left(\varphi_{h_{1} h_{2}(s)} x, \varphi_{h_{2}(s)} a\right) \leq \varepsilon_{0} \text { for all }-n T-r \leq h_{2}(s) \leq n T+r .
$$

The triangle inequality and (ii) imply

$$
d\left(\varphi_{h_{1} h_{2}(s)} x, \varphi_{s} b\right) \leq 2 \varepsilon_{0} \text { for all }-n T-r \leq s \leq n T+r .
$$

Again by replacing $h_{3}(s)$ by $s$ we have

$$
d\left(\varphi_{h_{1} h_{2} h_{3}(s)} x, \varphi_{h_{3}(s)} b\right) \leq 2 \varepsilon_{0} \text { for all }-n T \leq s \leq n T .
$$

The triangle inequality and (iii) imply

$$
d\left(\varphi_{h(s)} x, \varphi_{s} y\right) \leq 3 \varepsilon_{0} \text { for all }-n T \leq s \leq n T,
$$

where $h=h_{1} h_{2} h_{3}$. Similarly we can show that

$$
d\left(\varphi_{\beta(s)} y, \varphi_{s} x\right) \leq 3 \varepsilon_{0} \text { for all }-n T \leq s \leq n T,
$$

where $\beta=\beta_{3} \beta_{2} \beta_{1}$. But $3 \varepsilon_{0} \leq \varepsilon$. Thus $(x, y) \in V_{n}$ and the proof is finished.

The following is an immediate consequence of the above lemma and the metrization lemma [9].

Lemma 32. There is a metric $\rho$ on $S^{+}$compatible with the relative topology of $S^{+}$such that

$$
N\left(\Delta ; 1 / 2^{n+1}\right) \subseteq V_{n} \subseteq N\left(\Delta ; 1 / 2^{n}\right)
$$

for $n \geq 1$.

Now consider the flow $\psi$ on $X$ defined by $\psi_{s}(x)=\varphi_{s T}(x)$ for all $x \in X$ (i.e., $\psi_{1}=\varphi_{T}$ ). It is well known that $\psi$ is also uniformly expansive on $X$ and $h(\psi)=\operatorname{Th}(\varphi)[5,1]$.

Proposition 33. $\bar{T}(\psi)=\lim _{t \rightarrow \infty}(1 / t) \log N_{\rho}\left((1 / 2)^{t}\right)$.

Proof. Let $E$ be any $(\overline{n+r, \varepsilon})$-tracing set of $S^{+}$(i.e., for any $x \in S^{+}$, there exist $e \in E$ and $\alpha \in \operatorname{Rep}(R)$ so that $d\left(\psi_{\alpha(s)} e, \psi_{s} x\right) \leq \varepsilon$ for all $-n-r \leq$ $s \leq n+r)$. Thus $d\left(\psi_{u} e, \psi_{\alpha^{-1}(u)} x\right) \leq \varepsilon$ for all $-n \leq s \leq n$, where $u=$ $\alpha(s)$. This means that $(e, x) \in V_{n}$. Hence $(e, x) \in N\left(\Delta ; 1 / 2^{n}\right)$ and therefore $N_{\rho}\left((1 / 2)^{n}\right) \leq \bar{T}_{n+\lambda}\left(S^{+}, \varepsilon\right)$. This implies that

$$
\lim _{n \rightarrow \infty} \frac{1}{n} \log N_{\rho}\left(\left(\frac{1}{2}\right)^{n}\right) \leq \lim _{n \rightarrow \infty} \frac{1}{n} \log \bar{T}_{n+\lambda}\left(S^{+}, \varepsilon\right) \leq \bar{T}\left(\psi \mid S^{+}\right) .
$$

Now suppose $F$ is a $(1 / 2)^{n}$-net of $S^{+}$. This means that for every $x \in S^{+}$, there exists $e \in F$ such that $(x, e) \in N\left(\Delta ; 1 / 2^{n}\right)$. Thus $(x, e) \in V_{n-1}$ and 
therefore $F$ is a $(\overline{n-1, \varepsilon})$-tracing set of $S^{+}$. This implies that $\bar{T}_{n-1}\left(S^{+}, \varepsilon\right) \leq$ $N_{\rho}\left((1 / 2)^{n}\right)$ and

$$
\bar{T}\left(\psi \mid S^{+}\right)=\lim _{n \rightarrow \infty} \frac{1}{n} \log \bar{T}_{n-1}\left(S^{+}, \varepsilon\right) \leq \lim _{n \rightarrow \infty} \frac{1}{n} \log N_{\rho}\left(\left(\frac{1}{2}\right)^{n}\right) .
$$

Therefore

$$
\bar{T}\left(\psi \mid S^{+}\right)=\lim _{n \rightarrow \infty} \frac{1}{n} \log N_{\rho}\left(\left(\frac{1}{2}\right)^{n}\right) .
$$

Using Lemma 28(iii) we are done.

Theorem 34. $\exists \lambda$ with $0<\lambda<1$ such that

$$
h(\varphi)=\lim _{t \rightarrow \infty} \frac{1}{t} \log N_{\rho}\left(\lambda^{t}\right) .
$$

Proof. Using Lemma 26 and Lemma 15 we have $\bar{T}(\psi)=2 T(\psi), T(\psi)=$ $h(\psi)$, and $h(\psi)=T h(\varphi)$. Thus $2 T h(\varphi)=\bar{T}(\psi)$. Proposition 33 implies that

$$
2 T h(\varphi)=\lim _{t \rightarrow \infty} \frac{1}{t} \log N_{\rho}\left(\left(\frac{1}{2}\right)^{t}\right) \text {. }
$$

Thus,

$$
h(\varphi)=\lim _{t \rightarrow \infty} \frac{1}{2 t T} \log N_{\rho}\left(\left(\frac{1}{2}\right)^{n}\right) .
$$

Taking $\lambda=(1 / 2)^{1 / 2 T}$, we have

$$
h(\varphi)=\lim _{t \rightarrow \infty} \frac{1}{2 t T} \log N_{\rho}\left(\lambda^{2 t T}\right) .
$$

This means that

$$
h(\varphi)=\lim _{t \rightarrow \infty} \frac{1}{t} \log N_{\rho}\left(\lambda^{t}\right),
$$

and the proof is finished.

Theorem 34 is a key to define the topological entropy on foliations.

\section{REFERENCES}

1. R. Bowen, Entropy for group endomorphisms and homogeneous spaces, Trans. Amer. Math. Soc. 153 (1970), 401-414.

2. __ Periodic orbits for hyperbolic flows, Amer. J. Math. 94 (1972), 1-30.

3. __ Entropy-expansive maps, Trans. Amer. Math. Soc. 164 (1972), 323-331.

4. __ Topological entropy and Axiom A, Proc. Sympos. Pure Math., vol. 14, Amer. Math. Soc., Providence, R. I., 1970, pp. 23-41.

5. R. Bowen and P. Walters, Expansive one-parameter flows, J. Differential Equations 12 (1972), 180-193.

6. R. Bowen, Symbolic dynamics for hyperbolic flows, Amer. J. Math. 95 (1973), 429-460.

7. J. Franke and J. Selgrade, Hyperbolic and chain recurrence, J. Differential Equations 26 (1977), 27-36. 
8. Soc. 60 (1976), 309-316.

9. J. L. Kelley, General topology, Van Nostrand, Princeton, N.J., 1955.

10. H. B. Keynes and M. Sears, Real-expansive flows and topological dimensions, Ergodic Theory Dynamical Systems 1 (1981), 179-195.

11. W. Reddy, Expansive cannonical coordinates are hyperbolic, Topology Appl. 15 (1983), 205-210.

12. S. Smale, Differentiable dynamical systems, Bull. Amer. Math. Soc. 73 (1967), 747-817.

13. R. Thomas, Entropy of expansive flows, Ergodic Theory Dynamical Systems 7 (1987), 611-625.

14. _ Topological stability: some fundamental properties, J. Differential Equations 59 (1985), 103-122.

15. _ Stability properties of one parameter flows, Proc. London Math. Soc. (3) 45 (1982), 479-505.

16. H. Whitney, Regular families of curves, Ann. of Math. 34 (1933), 244-270. 22030

Department of Mathematical Sciences, George Mason University, Fairfax, Virginia

Current address: Embry-Riddle Aeronautical University, Prescott, Arizona 86301 